\title{
可见光促进的酰胺氮自由基参与的分子内氢胺化反应
}

\author{
余晓叶 周帆陈加荣* 肖文精 \\ (农药与化学生物学教育部重点实验室 华中师范大学化学学院 武汉 430079)
}

\begin{abstract}
摘要 报道了可见光促进的邻烯基取代苯甲酰胺的分子内自由基氢胺化反应. 在该反应中, 通过脱质子光致电子转移 策略实现了氮一氢键的直接催化活化来产生氮自由基, 随后通过氮自由基与烯烃的加成来实现氢胺化反应. 该反应以 优异的收率合成了一系列具有潜在生理活性的 3,4-二氢异喹啉酮衍生物. 该反应体系利用有机染料催化剂 EosinY Na 作为光氧化还原催化剂、廉价易得的 $\mathrm{NaOH}$ 作为碱试剂、反应条件温和、操作简单、且官能团兼容性好. 另外, 该反 应在放大量到克级规模、或者利用太阳光作为光源，均能以优异的收率得到相应目标化合物. 因此，这类反应为 3,4-二 氢异喹啉酮的合成提供了一个简便有效的方法.
\end{abstract}

关键词 可见光催化; 氮自由基; 氢胺化反应; 氮杂环; 3,4-二氢异哇啉酮

\section{Visible Light Photocatalytic $N$-Radical-based Intramolecular Hydroamination of Benzamides}

\author{
Yu, Xiaoye Zhou, Fan Chen, Jiarong* Xiao, Wenjing \\ (Key Laboratory of Pesticide \& Chemical Biology Ministry of Education and College of Chemistry, Central China Normal \\ University, Wuhan 430079)
}

\begin{abstract}
The 3,4-dihydroisoquinolinones are a privileged class of heterocyclic motifs and widely found in numerous biologically active compounds. Thus, the development of more efficient and practical methods for their synthesis is highly desirable. Traditional methods are typically focused on transition-metal catalyzed $\mathrm{C}-\mathrm{H}$ functionalization. Inspired by the recent process of the visible light photocatalytic generation and exploration of $N$-radicals in organic synthesis, our group in 2014 developed a visible light-induced photocatalytic strategy for direct conversion of the $\mathrm{N}-\mathrm{H}$ bonds of $\beta, \gamma$-unsaturated hydrazones into $N$-centred radicals for the first time, and used them in intramolecular radical hydroamination, enabling efficient synthesis of 4,5-dihydropyrazole derivatives. By employing suitable additives or changing reaction parameters, we also successfully achieved highly regioselective 6-endo $N$-radical cyclization and oxyamination reactions based on $N$-centred radicals, providing the valuable 1,6-dihydropyradazines, pyrazolines, and pyridazines in good yields. In the hope of extending such $N$-radical-mediated heterocycle synthesis further, we reported a transition-metal free and visible light photocatalytic $N$-radical-based intramolecular hydroamination of benzamides. The reaction provides a practical and efficient approach to various biologically important 3,4-dihydroisoquinolinones with generally high yields. Importantly, the continuous flow reaction could significantly shorten the reaction time and still give rise to satisfactory yield. The sunlight irradiation reaction and gram-scale reaction also highlighted the potential synthetic utility of this method. A general procedure for the reaction is as follows: EosinY Na (6.21 mg, $0.009 \mathrm{mmol}), \mathrm{NaOH}(14.4 \mathrm{mg}, 0.36 \mathrm{mmol})$, amide 1 (0.3 mmol) were dissolved in $\mathrm{MeOH}(6.0$ $\mathrm{mL}$ ), then, the resulting mixture was degassed via a 'freeze-pump-thaw' procedure (3 times). After that, the resulting mixture was stirred at a distance of $c a .5 \mathrm{~cm}$ from $3 \mathrm{~W}$ blue LEDs $(450 \sim 460 \mathrm{~nm})$ at room temperature until the starting amides were consumed as monitored by TLC analysis. After concentration in vacuo, the reaction residue was purified by flash chromatography on silica gel $[V($ petroleum ether $) / V($ ethyl acetate $)=5: 1 \sim 2: 1]$ directly to give the desired product.

Keywords visible light photocatalysis; $N$-radicals; hydroamination; $N$-heterocycles; 3,4-dihydroisoquinolinones
\end{abstract}

\section{1 引言}

3,4-二氢异喹啉酮骨架是许多具有生物活性的天然 产物以及药物活性分子的核心骨架(图 1) ${ }^{[1]}$. 例如，帕洛 诺司琼 A 是一类新型 5-HT3 受体阻滞剂, 可用于缓解或 预防化疗引起的急性呕吐, 恶心. 因此, 发展 3,4-二氢
喹啉酮的合成方法一直受到广大化学家们的关注.

目前, 对于 3,4-二氢喹啉酮这类化合物的合成有较 多文献报道, 但是大多数方法是基于金属催化的偶联和 环化反应等策略 ${ }^{[2]}$. 例如, 2011 年, Fagnou 小组 ${ }^{[2]}$ 报道了 在 $\left[\mathrm{Cp}^{*} \mathrm{RhCl}_{2}\right]_{2}$ 催化剂的作用下, 利用氮甲氧基苯甲酰

* E-mail: chenjiarong@mail.ccnu.edu.cn; Fax: 027-67862041

Received July 28, 2016; published September 20, 2016.

Supporting information for this article is available free of charge via the Internet at http://sioc-journal.cn.

Project supported by the National Natural Science Foundation of China (Nos. 21272087, 21472058, 21472057, and 21232003), and the Youth Chen-Guang Project of Wuhan (No. 2015070404010180).

项目受国家自然科学基金(Nos. 21272087, 21472058, 21472057, and 21232003)和武汉市青年科技晨光计划项目(No. 2015070404010180)资助. 
胺与炔烃的偶联环化反应来高效构建异喹啉酮类化合 物. 在该反应中，底物苯甲酰胺中的氮甲氧基部分同时 充当氧化剂和导向基作用. 同年, Glorius 小组 ${ }^{[2 \mathrm{~d}]}$ 发展了 $\mathrm{Rh}$ 催化的氮烷氧基苯甲酰胺和烯烃的偶联环化反应， 合成了一系列取代的 3,4-二氢异喹啉酮. 2013 年, 王从 洋课题组 ${ }^{[2 \mathrm{e}]}$ 报道了在高温条件下, $\mathrm{Re} / \mathrm{Mg}$ 共催化的苯甲 酰胺与炔烃的形式上的 $[4+2]$ 环化反应, 以优异的立体 选择性(cis-/trans) 和收率得到了一系列多取代和结构多 样的 3,4-二氢喹啉酮. 尽管这些方法为构建 3,4-二氢取 代异喹啉酮类化合物提供了有效的途径, 但是, 寻求更 为温和、甚至无过渡金属参与的绿色合成方法仍然是十 分必要的.

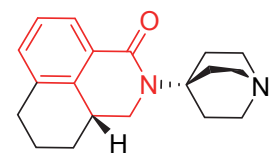

帕洛诺司琼A

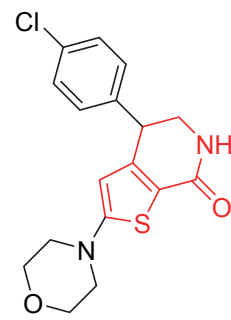

B

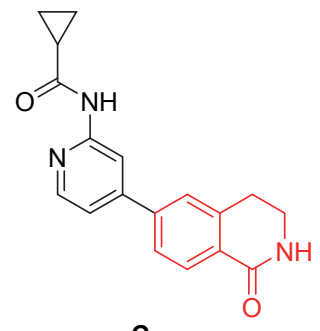

C

图 1 一些含有 3,4-二氢异喹啉酮骨架的生物活性分子

Figure 1 Selected examples of bioactive compounds containing 3,4-dihydroisoquinolinone motif

氮自由基化学是有机合成中一个重要的研究领域, 长期以来一直受到众多化学家们的关注 ${ }^{[3]}$. 其中, 氮自 由基与烯烃的加成环化策略为构建多官能化的氮杂环 化合物提供了强有力的方法. 氮一氢键是产生氮自由基 最为直接的前体. 然而, 由于大多数氮-氢键具有较高 的化学键裂解能, 把氮一氢键直接转化为氮自由基比较 困难. 通常需要使用具有一定毒性的 ${ }^{t} \mathrm{Bu}_{3} \mathrm{SnH}$ 等自由基 引发剂或当量的氧化剂 ${ }^{[4]}$; 另外, 也可以把氮一氢键预先 转化为较弱的氮一卤键、氮一氧键等, 再进一步转化为氮 自由基. 此外, 由于氮自由基本身活性较高, 容易从反 应体系中篗取氢原子重新生成氮一氢键. 这些挑战都阻 碍了氮自由基化学的快速发展.

近年来, 可见光促进的光氧化还原催化策略因其条 件温和、反应操作简单、绿色环保等优点而受到了广大 化学家们的关注, 在有机合成领域得到了广泛的应用 ${ }^{[5]}$. 在可见光促进的反应中, 本质上大多是基于光氧化还原 催化策略来产生碳自由基、杂原子自由基或者离子等活
泼中间体，从而实现碳一碳、碳-氮、碳-磷、碳-氧、碳卤等化学键的构建. 随着可见光催化领域发展, 该催化 策略为氮自由基的温和产生提供了新的方法，也为氮自 由基的新反应设计和含氮杂环的合成提供了重要平

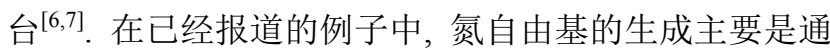
过氮一氧键或氮一卤键的还原断裂实现，缺少原子经济 性. 基于我们长期在光化学和自由基化学方面的研究兴 趣，我们课题组发展了 “脱质子光致电子转移” 策略，并 首次实现了可见光促进的 $\beta, \gamma$-不饱和腙的氮一氢键的直 接催化活化来产生氮自由基，发展了在温和条件下的分 子内氮自由基的氢胺化反应，合成了一系列 4,5-二氢吡 唑类化合物 ${ }^{[8]}$. 通过加入 TEMPO 添加剂以及催化体系 的合理设计, 还以较高的区域选择性实现了 6-endo 氮自 由基环化和羟胺化反应, 以较好的收率得到了 1,6-二氢 哒嗪、二氢吡唑等氮杂环化合物 ${ }^{[9]}$. 在本文中, 我们报道 了把脱质子光致电子转移策略进一步成功应用到邻烯 基取代苯甲酰胺的氮一氢键的直接催化活化来产生酰胺 氮自由基，并发展了温和条件下分子内氢胺化反应，以 优异的收率合成了一系列 3,4-二氢异喹啉酮类化合物.

\section{2 结果与讨论}

\section{1 反应条件的优化}

首先, 我们以邻烯基取代苯甲酰胺 1a 为模板底物, 以 $\left\{\operatorname{Ir}\left[\mathrm{dF}\left(\mathrm{CF}_{3}\right) \mathrm{PPy}\right]_{2} \mathrm{dtbbpy}\right\} \mathrm{PF}_{6}$ 为光催化剂, 以 $\mathrm{NaOH}$ 为 碱, 利用 $\mathrm{MeOH}$ 作为溶剂, 反应在 $12 \mathrm{~h}$ 后以 $92 \%$ 的 $\mathrm{GC}$ 收率得到相应的 $N$-甲氧基-4-苯基-3,4-二氢异喹啉酮产 物(表 1, Entry 1). 随后, 我们考察了一系列常用的光催 化剂. 当选用染料催化剂 EosinY $\mathrm{Na}$ (表 1, Entry 4)时, 我们以 $96 \%$ GC 收率和 $92 \%$ 的分离收率得到目标化合 物. 以 EosinY $\mathrm{Na}$ 作为最优催化剂, 我们随后对不同碱 试剂进行了篮选. 系列结果表明, 当采用 $\mathrm{NEt}_{3}$ (表 1 , Entry 5)作碱时, 反应几乎不发生, 而当选用 $\mathrm{NaHCO}_{3}$ 和 $\mathrm{K}_{2} \mathrm{CO}_{3}$ (表 1, Entries 6 7) 为碱时, 反应仍能以较高的 $\mathrm{GC}$ 收率生成目标化合物. 其他常用的碱, 如 $\mathrm{NaOAc}$ 和 $\mathrm{Cs}_{2} \mathrm{CO}_{3}$ 都只能给出中等的收率(表 1, Entries 8 9). 因 此, 我们仍然选择 $\mathrm{NaOH}$ 作为最佳碱试剂, 对反应溶剂 进行了优化(表 1, Entries 10～13). 结果表明, 溶剂对该 反应影响较大. 例如, 采用 $\mathrm{CHCl}_{3}$ 和 $\mathrm{CH}_{3} \mathrm{CN}$ 作溶剂时, 反应没有给出更好的结果(表 1, Entries 10 11); 选用 $\mathrm{DMF}$ 作溶剂时, 反应几乎不发生 (表 1, Entry 13). 因此, $\mathrm{MeOH}$ 仍然是最佳溶剂(表 1, Entry 4). 最终, 我们确立 了最优的反应条件, 即以 EosinY $\mathrm{Na}$ 作为光催化剂, $\mathrm{NaOH}$ 作碱, $\mathrm{MeOH}$ 作溶剂. 另外, 我们也尝试了严格的 控制实验. 实验结果表明可见光照射、光催化剂以及碱 试剂对该反应都是必需的(表 1, Entries 14 16).

\section{2 底物普适性的考察}

确立了最优反应条件后, 我们首先对底物氮原子上 
表 1 可见光促进的氮自由基的分子内氢胺化反应的条件篮选

Table 1 Optimization of reaction conditions for visible light-induced $\mathrm{N}$-radical-based intramolecular hydroamination

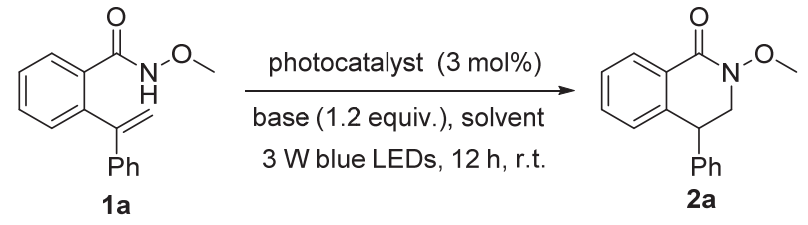

\begin{tabular}{|c|c|c|c|c|}
\hline Entry $^{a}$ & Photocatalyst & Base & Solvent & Yield $^{b} / \%$ \\
\hline 1 & $\left\{\operatorname{Ir}\left[\mathrm{dF}\left(\mathrm{CF}_{3}\right) \mathrm{PPy}\right]_{2} \mathrm{dtbbpy}\right\} \mathrm{PF}_{6}$ & $\mathrm{NaOH}$ & $\mathrm{MeOH}$ & 92 \\
\hline 2 & $\mathrm{Ru}(\text { bpy })_{3} \mathrm{Cl}_{2} \cdot 6 \mathrm{H}_{2} \mathrm{O}$ & $\mathrm{NaOH}$ & $\mathrm{MeOH}$ & 94 \\
\hline 3 & $N$-Me-9-Mes-Acr-ClO & $\mathrm{NaOH}$ & $\mathrm{MeOH}$ & trace \\
\hline 4 & EosinY $\mathrm{Na}$ & $\mathrm{NaOH}$ & $\mathrm{MeOH}$ & $96(92)$ \\
\hline 5 & EosinY Na & $\mathrm{NEt}_{3}$ & $\mathrm{MeOH}$ & trace \\
\hline 6 & EosinY Na & $\mathrm{NaHCO}_{3}$ & $\mathrm{MeOH}$ & 89 \\
\hline 7 & Eosin $\mathrm{Y} \mathrm{Na}$ & $\mathrm{K}_{2} \mathrm{CO}_{3}$ & $\mathrm{MeOH}$ & 90 \\
\hline 8 & EosinY $\mathrm{Na}$ & $\mathrm{NaOAc}$ & $\mathrm{MeOH}$ & 48 \\
\hline 9 & EosinY Na & $\mathrm{Cs}_{2} \mathrm{CO}_{3}$ & $\mathrm{MeOH}$ & 55 \\
\hline 10 & EosinY $\mathrm{Na}$ & $\mathrm{NaOH}$ & $\mathrm{CHCl}_{3}$ & 10 \\
\hline 11 & EosinY $\mathrm{Na}$ & $\mathrm{NaOH}$ & $\mathrm{CH}_{3} \mathrm{CN}$ & 72 \\
\hline 12 & EosinY $\mathrm{Na}$ & $\mathrm{NaOH}$ & THF & 5 \\
\hline 13 & EosinY $\mathrm{Na}$ & $\mathrm{NaOH}$ & DMF & trace \\
\hline $14^{c}$ & EosinY Na & $\mathrm{NaOH}$ & $\mathrm{MeOH}$ & trace \\
\hline $15^{d}$ & - & $\mathrm{NaOH}$ & $\mathrm{MeOH}$ & 0 \\
\hline $16^{e}$ & EosinY $\mathrm{Na}$ & - & $\mathrm{MeOH}$ & trace \\
\hline
\end{tabular}

${ }^{a}$ Reaction conditions: 1a $(0.1 \mathrm{mmol})$, photocatalyst $(3 \mathrm{~mol} \%)$, base $(0.12$ $\mathrm{mmol})$, solvent $(2.0 \mathrm{~mL}), 3 \mathrm{~W}$ blue LEDs, at room temperature; ${ }^{b}$ Determined by GC with biphenyl as an internal standard, yields of isolated products are shown in parentheses; ${ }^{c}$ Without visible light irradiation. ${ }^{d}$ without catalyst. ${ }^{e}$ Without base.

的取代基进行了考察. 当氮原子上的取代基由甲氧基变 为苦氧基时, 该反应能以 75\%的分离收率得到目标产物 2b. 当氮原子的取代基为苯基时, 该反应不能发生. 随 后, 我们考察了与烯烃相连的苯环上的取代基电性和取 代模式对反应的影响. 结果表明, 无论在芳环的对位引 入给电子基团 $(\mathrm{OMe}, 1 \mathrm{~d})$ 或吸电子基团 $(\mathrm{F}, 1 \mathrm{e})$, 还是在间 位引入两个甲基(1f)或单个氯取代基(1g)时, 反应均能 以较好的收率得到目标化合物 $\mathbf{2 d} \sim \mathbf{2 g}$. 当杂芳环取代 时, 该反应也能顺利进行, 以 $93 \%$ 的收率得到氢胺化产 物 $2 \mathrm{~h}$. 遗憾的是, 当将芳基改变为烷基, 如甲基(1i)或 环己基 $(\mathbf{1 j})$ 时, 反应不发生, 且几乎定量回收原料. 另 外, 当苯甲酰胺的苯环上引入不同电性的取代基时, 如 氯(1k), 甲氧基(11), 反应也都能以中等到较好的收率得 到产物 $2 \mathrm{k}$ 和 $2 \mathrm{l}$. 对于底物中的烯烃部分, 遗憾的是, 我 们这一方法学目前只适用于末端烯烃. 对于非末端烯 烃, 推测可能是由于位阻原因导致反应难以发生, 需要
进一步的反应体系优化才能有望解决这一问题. 另外, 底物 $\mathrm{N}$ 原子上的烷氧基取代基对该反应也是至关重要 的，如甲基取代时，反应并不能发生 ${ }^{[10]}$.

表 2 可见光促进的氮自由基的氢胺化反应的普适性考察

Table 2 Scope of the visible light-induced $N$-radical-based intramolecular hydroamination ${ }^{a}$<smiles>[R]ONC(=O)c1ccc([R])cc1C(=C)Br</smiles>

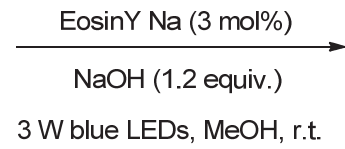

1<smiles>[R]ON1CC(Br)c2cc([R])ccc2C1=O</smiles><smiles>CON1CC(c2ccccc2)c2ccccc2C1=O</smiles>

2a $89 \%$<smiles>O=C1c2ccccc2C(c2ccccc2)CN1OCc1ccccc1</smiles>

2b $75 \%$<smiles>O=C1c2ccccc2C(c2ccccc2)CN1c1ccccc1</smiles>

2c no reaction<smiles>CON1CC(c2ccc(OC(C)(C)C)cc2)c2ccccc2C1=O</smiles><smiles>CON1CC(c2ccc(F)cc2)c2ccccc2C1=O</smiles><smiles>CCON1CC(c2cc(C)cc(C)c2)c2ccccc2C1=O</smiles><smiles>CON1CC(c2cccc(Cl)c2)c2ccccc2C1=O</smiles>

2g $87 \%$<smiles>CON1CC(C2CCCCC2)c2ccccc2C1=O</smiles>

2j no reaction<smiles>CON1CC(c2cccs2)c2ccccc2C1=O</smiles>

2h $93 \%$<smiles>CON1CC(C)c2ccccc2C1=O</smiles>

2i no reaction<smiles>CCON1CC(c2ccccc2)c2ccc(OC)cc2C1=O</smiles>

${ }^{a}$ Reaction conditions: $1(0.3 \mathrm{mmol})$, EosinY Na $(3 \mathrm{~mol} \%), \mathrm{NaOH}(0.36 \mathrm{mmol})$ in $\mathrm{MeOH}(6.0 \mathrm{~mL})$ under argon atmosphere for $24 \mathrm{~h}$, isolated yield.

为了进一步展示该合成方法的潜在应用性，我们尝 试将该反应放大量到克级规模(图 2). 在标准条件下，选 用 $\mathbf{1 f}$ 为底物，经过 $24 \mathrm{~h}$, 反应仍能以 $95 \%$ 的收率得到目 
标产物 2f.<smiles>C=C(c1cc(C)cc(C)c1)c1ccccc1C(=O)NOC</smiles>

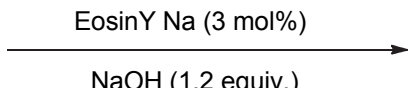

$3 \mathrm{~W}$ blue LEDs, $\mathrm{MeOH}$, r.t., $24 \mathrm{~h}$ 1f, $1.02 \mathrm{~g}$<smiles>CON1CC(c2cc(C)cc(C)c2)c2ccccc2C1=O</smiles>

2f, $95 \%$ yield<smiles>C=C(c1ccccc1)c1ccccc1C(=O)NOC</smiles>

1a, $0.2 \mathrm{mmol}$<smiles>CON1CC(c2ccccc2)c2ccccc2C1=O</smiles>

2a, 94\% yield

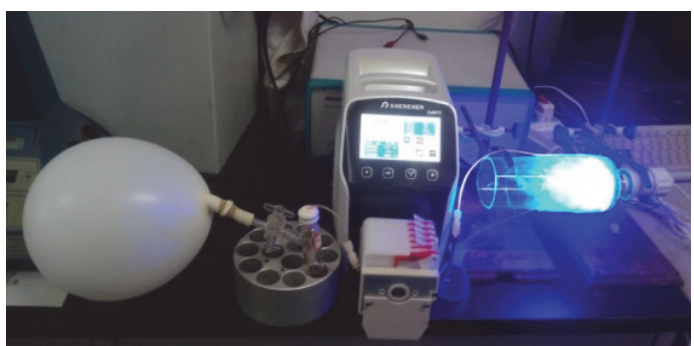

图 4 连续流动相反应

Figure 4 Continuous flow reaction<smiles>CCON1CC(c2ccccc2)c2ccccc2C1=O</smiles>

2a, $0.3 \mathrm{mmol}$

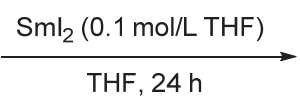

THF, $24 \mathrm{~h}$<smiles>C=C(c1ccccc1)c1ccccc1C(=O)NOC</smiles>

1a, $0.2 \mathrm{mmol}$<smiles>CON1CC(c2ccccc2)c2ccccc2C1=O</smiles>

2a, $88 \%$ yield
EosinY $\mathrm{Na}(3 \mathrm{~mol} \%)$ $\mathrm{NaOH}$ (1.2 equiv.), $\mathrm{MeOH}$, r.t., $3 \mathrm{~h}$
图 3 太阳光反应

Figure 3 Sunlight irradiation reaction

最近，连续流反应技术也被广泛用于光化学合成 中. 与传统的间歇反应(batch reaction)相比, 表现出了极 大的优势 ${ }^{[11]}$. 因此, 我们也尝试将模板反应放置在连续 流动光反应装置中进行, 大大地缩短了反应时间, 反应 $1 \mathrm{~h}$ 内即可完成并给出了高达 94\%的收率(图 4).

鉴于一些内酰胺类化合物在医药上的广泛应用, 我 们还尝试脱去氮原子上的甲氧基来构建二级内酰胺类 化合物. 参考文献方法 ${ }^{[12]}$, 当使用 $\mathrm{SmI}_{2}$ 作为还原剂时, 在 THF 溶剂中, $24 \mathrm{~h}$ 后我们可以顺利地以 $71 \%$ 的收率得 到 4-苯基异喹啉酮化合物 3 (图 5).

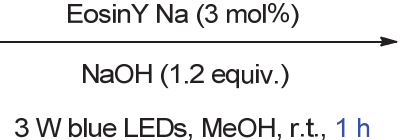

$3 \mathrm{~W}$ blue LEDs, $\mathrm{MeOH}$, r.t., 1 h 
基与烯烃部分发生分子内自由基加成反应, 生成碳自由 基中间体 III. 中间体 III 被还原态的光催化剂单电子还 原生成碳负离子 IV, 其发生进一步的质子化过程生成 最终的氢胺化产物 2a; 同时, 重新生成基态的光催化剂 从而完成光催化循环.

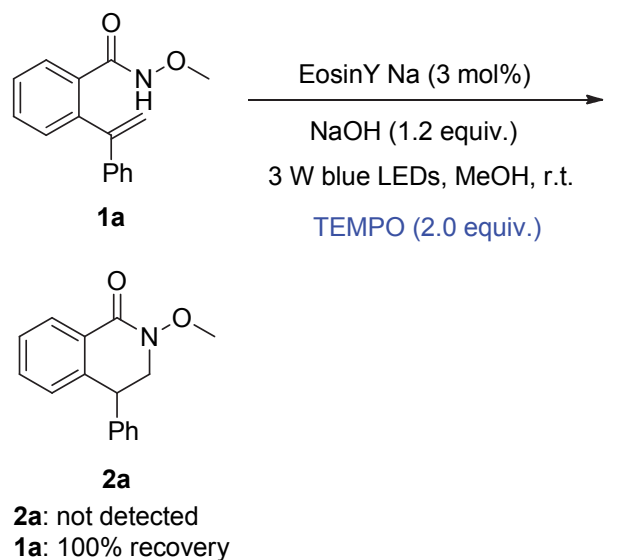

图 6 自由基捕获实验

Figure 6 Radical trapping experiment

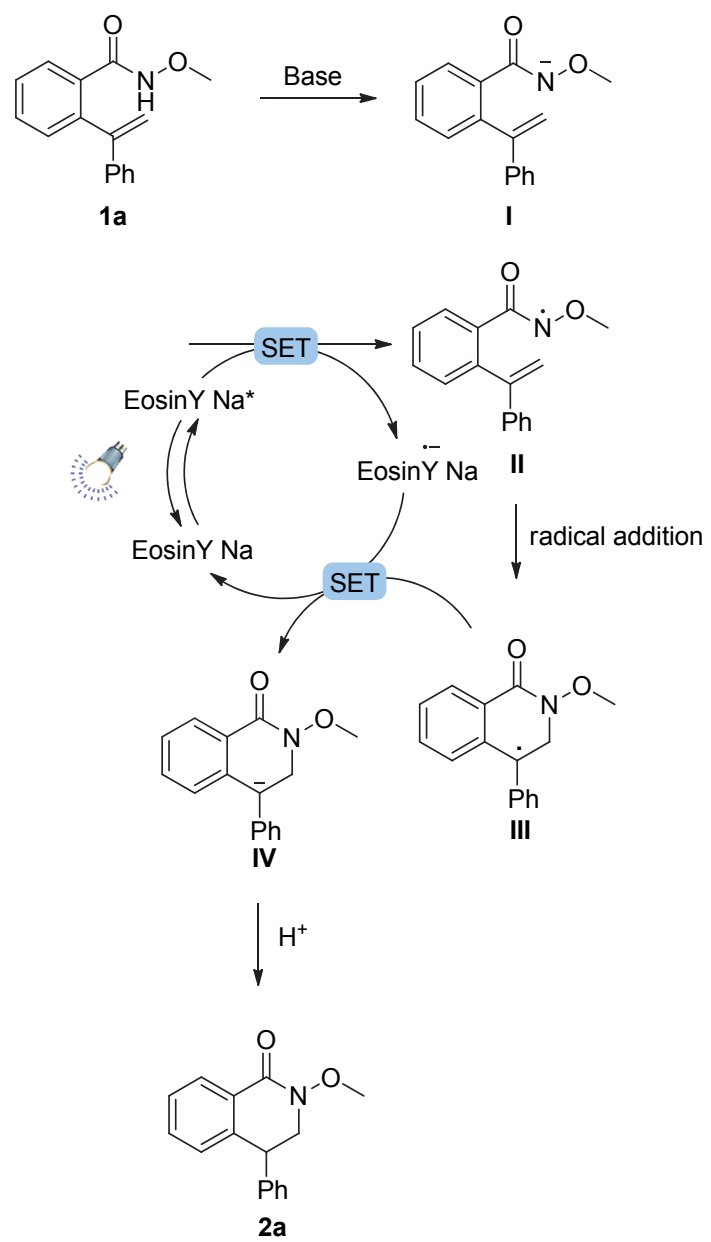

图 7 可能的反应机理

Figure 7 Proposed mechanism

\section{4 结论}

基于前期发展的脱质子光致电子转移策略，我们实 现了苯甲酰胺氮一氢键的直接催化活化来产生氮自由基, 并发展了温和条件下氮自由基的分子内氢胺化反应. 以 优异的收率构建了一系列取代 3,4-二氢异喹啉酮衍生 物. 该方法学可以放大量到克级规模或者使用太阳光作 为光源, 另外, 利用连续流反应技术可以大大地缩短反 应时间.

\section{5 实验部分}

可见光促进的酰胺氮自由基参与的分子内氢胺化 反应的实验方法: 向 $10 \mathrm{~mL}$ 干燥的 Schlenk 管中, 依次 加入底物 $1 \mathrm{a}(75.9 \mathrm{mg}, 0.3 \mathrm{mmol})$, EosinY $\mathrm{Na}(6.21 \mathrm{mg}$, $0.009 \mathrm{mmol}), \mathrm{NaOH}(14.4 \mathrm{mg}, 0.36 \mathrm{mmol}), \mathrm{MeOH}(6.0$ $\mathrm{mL}$ ), 通过 “freeze-pump-thaw” 的操作, 将反应体系中 的氧气除去. 随后, 将反应体系放在 $3 \mathrm{~W}$ blue LEDs 照 射下室温搅拌 $24 \mathrm{~h}$. 反应结束后, 减压浓缩后得到的混 合物进行柱层析分离纯化(石油醚/乙酸乙酯, $V / V=5$ : 1 2: 1), 以 $89 \%$ 的分离收率得到最终目标产物 2a. 2a 无色油状物(67.6 mg, 89\% yield); ${ }^{1} \mathrm{H}$ NMR (400 MHz, $\left.\mathrm{CDCl}_{3}\right) \delta: 8.21 \sim 8.19(\mathrm{~m}, 1 \mathrm{H}), 7.39 \sim 7.36(\mathrm{~m}, 2 \mathrm{H})$, $7.31 \sim 7.24(\mathrm{~m}, 3 \mathrm{H}), 7.16 \sim 7.13(\mathrm{~m}, 2 \mathrm{H}), 6.95 \sim 6.92(\mathrm{~m}$, $1 \mathrm{H}), 4.45(\mathrm{t}, J=6.4 \mathrm{~Hz}, 1 \mathrm{H}), 4.11 \sim 4.07(\mathrm{~m}, 1 \mathrm{H}), 3.91 \sim$ $3.87(\mathrm{~m}, 1 \mathrm{H}), 3.69(\mathrm{~s}, 3 \mathrm{H}) ;{ }^{13} \mathrm{C} \mathrm{NMR}\left(100 \mathrm{MHz}, \mathrm{CDCl}_{3}\right) \delta$ : $162.8,140.2,139.6,132.3,128.6,128.5,128.2,128.1$, $127.5,127.4,127.3,61.6,54.8,45.3$; IR (KBr) v: 1633, 1411, 1089, 1012, $904 \mathrm{~cm}^{-1}$; HRMS (EI) $m / z:[\mathrm{M}+\mathrm{H}]^{+}$ calcd for $\mathrm{C}_{16} \mathrm{H}_{16} \mathrm{NO}_{2} 254.1181$, found 254.1174.

\section{References}

[1] (a) Goldberg, D. US 2006276496, 2006 [Chem. Abstr. 2006, 146, 1286266]. (b) Goldberg, D. US 2010190773, 2010 [Chem. Abstr. 2006, 146, 1286266]. (c) Freeze, B. S. WO 20120051410, 2014 [Chem. Abstr. 2012, 156, 573694]. (d) Freeze, B. S. US 2015148334, 2015 [Chem. Abstr. 2012, 156, 573694]. (e) Zhang, L.; Wang, C.; Han, J.; Huang, Z. B.; Zhao, Y. J. Org. Chem. 2016, 81, 5256. (f) Welsch, M. E.; Snyder, S. A.; Stockwell, B. R. Curr. Opin. Chem. Biol. 2010, 14, 347.

[2] For selected reviews, see: (a) Zhao, J.; Zhang, Q. Acta Chim. Sinica 2015，73，1235. (赵金钵，张前，化学学报, 2015, 73, 1235.) (b) Yuan, J.-W.; Liu, C.; Lei, A.-W. Chem. Commun. 2015, 51, 1394. For selected examples, see: (c) Guimond, N.; Gorelsky, S. I.; Fagnou, K. J. Am. Chem. Soc. 2011, 133, 6449. (d) Rakshit, S. Grohmann, C.; Besset, T.; Glorius, F. J. Am. Chem. Soc. 2011, 133, 2350. (e) Tang, Q.; Xia, D.; Jin, X.; Zhang, Q.; Sun, X. Q.; Wang, C. J. Am. Chem. Soc. 2013, 135, 4628. (f) Wodrich, M. D.; Ye, B.; Gonthier, J. F.; Corminboeuf, C.; Cramer, N. Chem. Eur. J. 2014, 20, 15409. (g) Sivakumar, G.; Vijeta, A.; Jeganmohan, M. Chem. Eur. J. 2016, 22, 5899. (h) Hao, X.-Q.; Du, C.; Zhu, X.; Li, P.-X.; Zhang, J.-H.; Niu, J.-L.; Song, M.-P. Org. Lett. 2016, 18, 3610.

[3] For selected reviews on the $N$-radical chemistry, see: (a) Zard, S. Z.; Chem. Soc. Rev. 2008, 37, 1603. (b) Quiclet-Sire, B.; Zard, S. Z. Beilstein J. Org. Chem. 2013, 9, 557. (c) Hioe, J.; Šakić, D.; Vrček, V.; Zipse, H. Org. Biomol. Chem. 2015, 13, 157.

[4] For selected examples, see: (a) Sherman, E. S.; Chemler, S. R.; Tan, T. B.; Gerlits, O. Org. Lett. 2004, 6, 1573. (b) Sherman, E. S.; Fuller, P. H.; Kasi, D.; Chemler, S. R. J. Org. Chem. 2007, 72, 3896. (c) 
Zeng, W.; Chemler, S. R. J. Am. Chem. Soc. 2007, 129, 12948. (d) Zhu, X.; Wang, Y.-F.; Ren, W.; Zhang, F.-L.; Chiba, S. Org. Lett. 2013, 15, 3214. (e) Zhu, M.-K.; Chen, Y.-C.; Loh, T. P. Chem. Eur. J. 2013, 19, 5250. (f) Duan, X.-Y.; Zhou, N.-N.; Fang, R.; Yang, X.-L.; Yu, W.; Han, B. Angew. Chem. Int. Ed. 2014, 53, 3158. (g) Duan, X. Y.; Yang, X. L.; Jia, P. P.; Zhang, M.; Han, B. Org. Lett. 2015, 17, 6022 .

[5] For selected reviews on the visible light photocatalysis, see: (a) Xuan, J.; Xiao, W.-J. Angew. Chem. Int. Ed. 2012, 51, 6828. (b) Shi, L.; Xia, W.-J. Chem. Soc. Rev. 2012, 41, 7687. (c) Prier, C. K.; Rankic, D. A.; Macmillan, D. W. Chem. Rev. 2013, 113, 5322. (d) Xi, Y.-M.; Yi, H.; Lei, A.-W. Org. Biomol. Chem. 2013, 11, 2387. (e) Dai, X.; Xu, X.; Li, X. Chin. J. Org. Chem. 2013, 33, 2046. (戴小 军, 许孝良, 李小年, 有机化学, 2013, 33, 2046.) (f) Schultz, D. M.; Yoon, T. P. Science 2014, 343, 985. (g) Xuan, J.; Zhang, Z.-G.; Xiao, W.-J. Angew. Chem. Int. Ed. 2015, 54, 15632. (h) Tan, F.; Xiao, W.-J. Acta Chim. Sinica 2015, 73, 85. (谭芬, 肖文精, 化学学 报, 2015, 73, 85.) (i) Karkas, M. D.; Porco Jr., J. A.; Stephenson, C. R. Chem. Rev. 2016, 116, 9683. (j) Romero, N. A.; Nicewicz, D. A. Chem. Rev. 2016, 116, 10075. (k) Chen, J.-R.; Hu, X.-Q.; Lu, L.-Q.; Xiao, W.-J. Acc. Chem. Res. 2016, doi: 10. 1021/acs. accounts. $6 \mathrm{~b} 00254$.

[6] For recent reviews, see: (a) Chen, J.-R.; Hu, X. Q.; Lu, L.-Q.; Xiao, W.-J. Chem. Soc. Rev. 2016, 45, 2044. (b) Xiong, T.; Zhang, Q. Chem. Soc. Rev. 2016, 45, 3069.

[7] For recent examples on the visible light-induced $N$-radical reactions, see: (a) Cecere, G.; Konig, C. M.; Alleva, J. L.; MacMillan, D. W. J. Am. Chem. Soc. 2013, 135, 11521. (b) Allen, L. J.; Cabrera, P. J.; Lee, M.; Sanford, M. S. J. Am. Chem. Soc. 2014, 136, 5607. (c) Musacchio, A. J.; Nguyen, L. Q.; Beard, G. H.; Knowles, R. R. J. Am. Chem. Soc. 2014, 136, 12217. (d) Song, L.; Zhang, L.; Luo, S.;
Cheng, J.-P. Chem. Eur. J. 2014, 20, 14231. (e) Kim, H.; Kim, T.; Lee, D. G.; Roh, S. W.; Lee, C. Chem. Commun. 2014, 50, 9273. (f) Qin, Q.; Yu, S. Org. Lett. 2015, 17, 1894. (g) Jiang, H.; An, X.; Tong, K.; Zheng, T.; Zhang, Y.; Yu, S. Angew. Chem. Int. Ed. 2015 , 54, 4055. (h) Miller, D. C.; Choi, G. J.; Orbe, H. S.; Knowles, R. R. J. Am. Chem. Soc. 2015, 137, 13492. (i) Davies, J.; Booth, S. G.; Essafi, S.; Dryfe, R. A. W.; Leonori, D. Angew. Chem. Int. Ed. 2015, 54, 14017. (j) Davies, J.; Svejstrup, T. D.; Fernandez Reina, D.; Sheikh, N. S.; Leonori, D. J. Am. Chem. Soc. 2016, 138, 8092. (k) Zhu, L.; Xiong, P.; Mao, Z.-Y.; Wang, Y.-H.; Yan, X.-M.; Lu, X.; Xu, H.-C. Angew. Chem. Int. Ed. 2016, 55, 2226. (1) Zhao, Y.; Huang, B.; Yang, C.; Xia, W. Org. Lett. 2016, 18, 3326. (m) Brachet, E.; Marzo, L.; Selkti, M.; König, B.; Belmont, P. Chem. Sci. 2016, 7, 5002.

[8] Hu, X.-Q.; Chen, J.-R.; Wei, Q.; Liu, F.-L.; Deng, Q.-H.; Beauchemin, A. M.; Xiao, W.-J. Angew. Chem. Int. Ed. 2014, 53, 12163.

[9] (a) Hu, X.-Q.; Qi, X.; Chen, J.-R.; Zhao, Q.-Q.; Wei, Q.; Lan, Y.; Xiao, W.-J. Nat. Commun. 2016, 7, 11188. (b) Hu, X.-Q.; Chen, J.; Chen, J.-R.; Yan, D.-M.; Xiao, W.-J. Chem. Eur. J. 2016, doi: 10. 1002/chem. 201602597.

[10] Please see the Supporting Information for more details.

[11] For selected reviews, see: (a) Knowles, J. P.; Elliott, L. D.; BookerMilburn, K. I. Beilstein J. Org. Chem. 2012, 8, 2025. (b) Su, Y; Straathof, N. J. W.; Hessel, V.; Noël, T. Chem. Eur. J. 2014, 20 , 10562. (c) Garlets, Z. J.; Nguyen, J. D.; Stephenson, C. R. Isr. J. Chem. 2014, 54, 351.

[12] Yang, G.-Q.; Shen, C.-R.; Zhang, W.-B. Angew. Chem. Int. Ed. 2012, 51,9141 .

[13] (a) Nicewicz, D. A.; Nguyen, T. M. ACS Catal. 2014, 4, 355. (b) Hari, D. P.; Konig, B. Chem. Commun. 2014, 50, 6688.

(Cheng, B.; Fan, Y.) 